\title{
Comparison of electromagnetically induced transparency schemes in semiconductor quantum dot structures: Impact of many-body interactions
}

\author{
Houmark-Nielsen, Jakob; Nielsen, Torben Roland; Mørk, Jesper; Jauho, Antti-Pekka
}

Published in:

Physical Review B Condensed Matter

Link to article, DOI:

10.1103/PhysRevB.79.115420

Publication date:

2009

Document Version

Publisher's PDF, also known as Version of record

Link back to DTU Orbit

Citation (APA):

Houmark-Nielsen, J., Nielsen, T. R., Mørk, J., \& Jauho, A-P. (2009). Comparison of electromagnetically induced transparency schemes in semiconductor quantum dot structures: Impact of many-body interactions. Physical Review B Condensed Matter, 79(11), 115420. https://doi.org/10.1103/PhysRevB.79.115420

\section{General rights}

Copyright and moral rights for the publications made accessible in the public portal are retained by the authors and/or other copyright owners and it is a condition of accessing publications that users recognise and abide by the legal requirements associated with these rights.

- Users may download and print one copy of any publication from the public portal for the purpose of private study or research.

- You may not further distribute the material or use it for any profit-making activity or commercial gain

- You may freely distribute the URL identifying the publication in the public portal 


\title{
Comparison of electromagnetically induced transparency schemes in semiconductor quantum dot structures: Impact of many-body interactions
}

\author{
J. Houmark, ${ }^{1, *}$ T. R. Nielsen, ${ }^{2}$ J. Mørk, ${ }^{2}$ and A.-P. Jauho ${ }^{1,3}$ \\ ${ }^{1}$ Department of Micro- and Nanotechnology-DTU Nanotech, Technical University of Denmark, Ørsteds Plads, \\ DK-2800 Kongens Lyngby, Denmark \\ ${ }^{2}$ Department of Photonics Engineering-DTU Fotonik, Technical University of Denmark, Ørsteds Plads, \\ DK-2800 Kongens Lyngby, Denmark \\ ${ }^{3}$ Department of Applied Physics, Helsinki University of Technology, P.O. Box 1100, 02015 HUT, Finland \\ (Received 27 November 2008; revised manuscript received 23 January 2009; published 16 March 2009)
}

\begin{abstract}
We investigate the impact of many-body interactions on group-velocity slowdown achieved via electromagnetically induced transparency in quantum dots using three different coupling-probe schemes (ladder, $V$, and $\Lambda$, respectively). We find that for all schemes many-body interactions have an important impact on the slow light properties. In the case of the $\Lambda$ and $V$ schemes, the minimum required coupling power to achieve slow light is significantly reduced by many-body interactions. $V$ type schemes are found to be generally preferable due to a favorable redistribution of carriers in energy space.
\end{abstract}

DOI: $10.1103 /$ PhysRevB.79.115420

PACS number(s): 78.67.Hc, 71.35.Cc

\section{INTRODUCTION}

Quantum dot (QD) based materials are promising for applications exploiting quantum coherence phenomena due to their atomlike properties and long dephasing times. ${ }^{1}$ They have been proposed to act as active media in devices for controlling the emission pattern of phased array antennas ${ }^{2}$ or in slow light based all-optical buffers. ${ }^{3}$

A particular physical effect that can be utilized for generating slow light is electromagnetically induced transparency (EIT). EIT refers to an artificially created spectral region of transparency in the middle of an absorption line due to the destructive quantum interference arising from two transitions in a three-level system. ${ }^{4,5}$ By virtue of the Kramers-Krönig relations such an absorption reduction is accompanied by a large positive slope of the refractive index which translates into a reduced group velocity in the vicinity of the resonance.

Very recently, the first experimental studies of EIT in QD systems have been performed ${ }^{6}$ where a coherent absorption dip in a coupling-probe experiment has been observed for an optically thin structure. Semiconductor QD based EIT schemes without real carrier excitations have been studied using models from atomic physics. ${ }^{3,7-9}$ Such EIT configurations involve pumping of intraband transitions whose wavelengths lie in the deep infrared, a regime for which high intensity laser operation is very difficult. Carrier-exciting schemes using interband coupling transitions therefore come into play. Recently, a theoretical description with the inclusion of many-body effects for a solid-state QD EIT $\Lambda$ configuration has been reported..$^{10,11}$

Using carrier-exciting schemes one in effect addresses two types of quantum coherence phenomena, EIT, as well as coherent population oscillation ${ }^{12,13}(\mathrm{CPO})$. CPO is a fourwave mixing effect based on interference between the coupling and probe fields. One should therefore keep in mind that schemes involving carrier excitation generally would contain a mixture of the two effects. However, CPO can be ruled out by choosing a setup utilizing orthogonal polarization directions for the coupling and probe fields, thus preventing the possibility of interference.
Concerning the EIT effect, an inherent problem of the carrier-exciting scheme is that the carriers excited by the coupling field block the transitions via the Pauli blocking factor, effectively decreasing the strength of the transitions, making such configurations less attractive than those that do not excite carriers. In addition, the excited carriers modify the spectral properties of the system via their mutual Coulomb interaction. Such effects cannot be accounted for in the noninteracting model. The work presented in Refs. 10 and 11 addresses the many-body aspects of a carrier-exciting $\Lambda$ configuration in a transient regime. The study of pulse propagation in a semiconductor slow light medium would generally involve solving the coupled Maxwell-Bloch equations. However, under certain circumstances, an analysis of the steadystate properties of the semiconductor Bloch equations (SBEs) alone is adequate. In that sense the linear optical response extracted in this limit is directly linked to the propagation characteristics of a wave packet traveling in an optically thick QD system. Noncarrier-exciting schemes have only been studied with the inclusion of many-body effects in this limit on one occasion ${ }^{14}$ while studies of carrierexciting schemes have not, to the best of our knowledge, been discussed in the literature. The aim of this paper is to present a comparison between different EIT schemes, with and without carrier excitation, which can be realized in the same dot structure. We study the EIT generated slow light properties of InAs QDs by solving the generalized SBEs in the Hartree-Fock approximation. The slowdown capabilities of the ladder, $V$, and $\Lambda$ schemes (see Fig. 1) obtained in steady state are compared using two models: the atomic model where interactions are disregarded, and the interacting model where many-body effects are taken into account.

\section{THEORETICAL MODEL}

The heterostructure under consideration consists of conical InAs quantum dots (radius of $9 \mathrm{~nm}$ and height of $3 \mathrm{~nm}$ ) residing on a 1.2-nm-thick wetting layer (WL), sandwiched between two slabs of GaAs. The electronic structure is cal- 


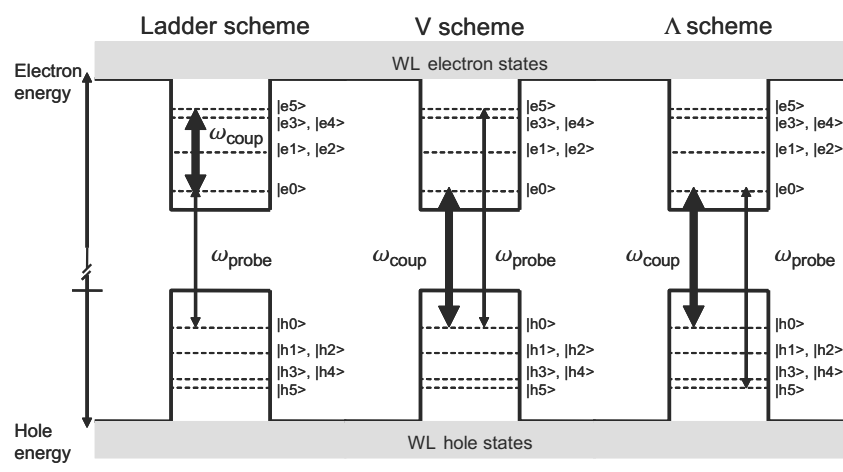

FIG. 1. Schematic quantum dot level structure and three EIT configurations. The frequency of the intense coupling field is denoted as $\omega_{\text {coup }}$ while the weak probe field is shown as $\omega_{\text {probe }}$. For illustrative purposes the figure has not been drawn to scale.

culated as the solution to the single-band Schrödinger equation for the envelope wave function in the effective-mass approximation. ${ }^{15}$ Using effective electron and hole masses $m_{e}=0.067 m_{0}$ and $m_{h}=0.15 m_{0}$, and a conduction-band/ valence-band offset $\mathrm{CBO}=705 \mathrm{meV}, \mathrm{VBO}=363 \mathrm{meV}$, we find six confined hole states (labeled $|h 0\rangle-|h 5\rangle$ ) as well as six confined electron states (labeled $|e 0\rangle-|e 5\rangle$ ), all doubly degenerate due to spin. Furthermore, the inherent rotational symmetry ensures complete degeneracy of the first and second excited as well as third and fourth excited states for both bands. For each band we also find the onset of a continuous set of delocalized states extending into the wetting layer. These WL states are treated as plane waves. The resulting energy-level structure, along with three different EIT schemes, is shown in Fig. 1.

The dipole selection rules allow for the realization of the three archetypical EIT schemes: ladder, $V$, and $\Lambda$. In the two latter cases, the coupling field excites an interband transition, resulting in optical pumping of the dot. We model experiments where a continuous-wave coupling field is irradiating a homogeneous ensemble of QDs. The steady-state system response is obtained by applying a weak probe pulse with a Gaussian envelope at times later than any other time scale of the system relative to the onset of the coupling field, such that transient effects may be neglected. In order to effectively utilize the slowdown of the light, as in an all-optical buffer, the probe field must propagate within the plane of the active medium. Assuming the QDs to lie in the $x-y$ plane, we take the probe polarization along the $x$ axis and the propagation direction along the $y$ axis. To completely rule out $\mathrm{CPO}$ effects we need orthogonal polarizations of the coupling and probe fields. For the $V$ and $\Lambda$ schemes the coupling field is polarized in the $y$ direction but in these situations we let the coupling field propagate perpendicular to the QD plane so that we can disregard propagation effects, e.g., attenuation, in the coupling field. In the ladder scheme the coupling field connects states $|e 0\rangle$ and $|e 5\rangle$, and is polarized in the growth direction of the QDs ( $z$ direction) and thus traveling in the QD plane. We disregard propagation effects as the coupling field is effectively connecting two empty states, thus rendering the transition transparent.

The interband dipole moments connecting $|e 0\rangle$ and $|h 5\rangle$ as well as $|e 5\rangle$ and $|h 0\rangle$ are nonzero even in the one-band effective-mass description. A detailed strain based eight-band $\mathbf{k} \cdot \mathbf{p}$ calculation ${ }^{16}$ shows, however, that these dipole moments are roughly a factor of 20 larger compared to the one-band result. As our focus is on the influence of the many-body interactions on the slowdown effects, we will therefore assume the k.p based results for the $\mu_{e 0 h 5}=10.24 e \AA$ and $\mu_{e 5 h 0}=10.14 e \AA$ dipole moments. The other relevant dipole moments are $\mu_{e 0 h 0}=15.55 e \AA$ and $\mu_{e 0 e 5}=2.79 e \AA$.

The linear optical response to the probe, i.e., the susceptibility $\chi(\omega)$, whose real and imaginary parts are related to refraction and absorption, respectively, is found from the macroscopic polarization $P(\omega)$ as $\chi(\omega)=\frac{P(\omega)}{\epsilon_{0} E_{p}(\omega)}$, where $\epsilon_{0}$ is the vacuum permittivity and $E_{p}(\omega)$ is the amplitude of the probe field.

The time resolved macroscopic polarization component in the direction of the probe field, $P(t)$, is computed from the microscopic polarizations according to semiclassical theory: ${ }^{17}$

$$
P(t)=\frac{1}{w}\left(N_{\mathrm{dot}} \sum_{i, j} \mu_{i j} P_{i j}(t)+\frac{1}{A} \sum_{\mathbf{k}}\left[\mu_{\mathbf{k}} P_{\mathbf{k}}(t)+\text { c.c. }\right]\right),
$$

where $P_{i j}$ and $P_{\mathbf{k}}$ are microscopic polarization components of localized dot states $(i, j)$ and diagonal interband polarization components of delocalized WL states $(\mathbf{k})$, respectively. In this treatment we disregard polarization components relating to transitions connecting dot and WL states. Dipole matrix elements between localized states are denoted $\mu_{i j}$, whereas $\mu_{\mathbf{k}}$ is the dipole moment relating to WL states. $N_{\mathrm{dot}}$ is the two-dimensional density of the dots in the WL plane, $A$ is the normalization area of the $\mathrm{WL}$, and $w$ is the thickness of the active region.

The microscopic polarizations are the off-diagonal components $\Psi_{\nu_{1} \nu_{2}}\left(\nu_{1} \neq \nu_{2}\right)$ of the reduced density matrix $\rho_{\nu_{1} \nu_{2}}$, where $\nu$ refers to either a QD state $i$ or a WL state $\mathbf{k}$. The time development of the polarizations are found by solving the SBEs in the Hartree-Fock approximation, see, e.g., Ref. 18, given (in the electron-electron picture for the sake of brevity) by

$$
\begin{gathered}
i \hbar \frac{\partial}{\partial t} \Psi_{\nu_{1} \nu_{2}}(t)-\left[\widetilde{\epsilon}_{\nu_{1}}(t)-\widetilde{\epsilon}_{\nu_{2}}(t)\right] \Psi_{\nu_{1} \nu_{2}}(t) \\
\quad-\left[n_{\nu_{2}}(t)-n_{\nu_{1}}(t)\right] \Omega_{\nu_{1} \nu_{2}}(t) \\
\quad-\sum_{\nu_{3} \neq \nu_{1}, \nu_{2}}\left[\Omega_{\nu_{1} \nu_{3}}(t) \Psi_{\nu_{3} \nu_{2}}(t)-\Psi_{\nu_{1} \nu_{3}}(t) \Omega_{\nu_{3} \nu_{2}}(t)\right] \\
\quad=i \hbar S_{\nu_{1} \nu_{2}}(t) \approx-i \hbar \gamma_{d} \Psi_{\nu_{1} \nu_{2}}, \\
i \hbar \frac{\partial}{\partial t} n_{\nu_{1}}(t)-\sum_{\nu_{3} \neq \nu_{1}}\left[\Omega_{\nu_{1} \nu_{3}}(t) \Psi_{\nu_{3} \nu_{1}}(t)-\Omega_{\nu_{3} \nu_{1}}(t) \Psi_{\nu_{1} \nu_{3}}(t)\right] \\
=i \hbar S_{\nu_{1} \nu_{1}}(t) \approx-i \hbar \gamma_{n r} n_{\nu_{1}}(t)-i \hbar \gamma_{c-c}\left[n_{\nu_{1}}(t)-f_{\nu_{1}}\left(\mu_{p}, T_{p}\right)\right] \\
-i \hbar \gamma_{c-p}\left[n_{\nu_{1}}(t)-f_{\nu_{1}}\left(\mu_{l}, T_{l}\right)\right],
\end{gathered}
$$

where

$$
\tilde{\epsilon}_{\nu}(t)=\epsilon_{\nu}+\sum_{\nu_{3} \nu_{4}}\left[V_{\nu \nu_{4} \nu_{3} \nu}-V_{\nu \nu_{4} \nu \nu_{3}}\right] \rho_{\nu_{3} \nu_{4}}(t),
$$




$$
\Omega_{\nu_{1} \nu_{2}}(t)=-e \mu_{\nu_{1} \nu_{2}} E(t)+\sum_{\nu_{3} \nu_{4}}\left[V_{\nu_{1} \nu_{4} \nu_{3} \nu_{2}}-V_{\nu_{1} \nu_{4} \nu_{2} \nu_{3}}\right] \rho_{\nu_{3} \nu_{4}}(t)
$$

are the Hartree-Fock renormalized single-particle energy and generalized Rabi frequency, respectively. $n_{\nu}$ is the diagonal component of the density matrix, i.e., $\rho_{\nu v}$. The term $-e \mu_{\nu_{1} \nu_{2}} E(t)$ is the electromagnetic field interaction in the dipole approximation, and the matrix elements of the Coulomb interaction are $\quad V_{\nu_{1} \nu_{2} \nu_{3} \nu_{4}}=\int \Phi_{\nu_{1}}^{*}(\mathbf{r}) \Phi_{\nu_{2}}^{*}\left(\mathbf{r}^{\prime}\right) e^{2} / \epsilon_{0} \epsilon_{b} \mid \mathbf{r}$ $-\mathbf{r}^{\prime} \mid \Phi_{\nu_{3}}\left(\mathbf{r}^{\prime}\right) \Phi_{\nu_{4}}(\mathbf{r}) d^{3} r d^{3} r^{\prime}$. Coulomb elements are found by approximating the numerically evaluated localized dot states by those of a harmonic oscillator, and $V_{\nu_{1} \nu_{2} \nu_{3} \nu_{4}}$ is then calculated following Refs. 19 and 20. For the situations considered here, screening effects are disregarded due to low WL densities.

Off-diagonal scattering terms $S_{\nu_{1} \nu_{2}}(t)$ are approximated by a temperature dependent effective dephasing rate $\gamma_{d}$,

$$
S_{\nu_{1} \nu_{2}}(t) \approx-\gamma_{d} \Psi_{\nu_{1} \nu_{2}} .
$$

Diagonal terms representing collision induced particle exchange processes are mimicked by a nonradiative recombination and a population relaxation toward quasiequilibrium Fermi-Dirac functions $f_{\nu},{ }^{21}$ determined by the charge-carrier density and temperature. The scattering rates are denoted as $\gamma_{c-c}$ and $\gamma_{c-p}$, representing carrier-carrier and carrier-phonon scattering, and the recombination rate is called $\gamma_{\mathrm{n}}$,

$$
\begin{aligned}
S_{\nu_{1} \nu_{1}}(t) \approx & -\gamma_{\mathrm{nr}} n_{\nu_{1}}(t)-\gamma_{c-c}\left[n_{\nu_{1}}(t)-f_{\nu_{1}}\left(\mu_{p}, T_{p}\right)\right] \\
& -\gamma_{c-p}\left[n_{\nu_{1}}(t)-f_{\nu_{1}}\left(\mu_{l}, T_{l}\right)\right] .
\end{aligned}
$$

Here $\mu$ and $T$ are the chemical potential and temperature of either the plasma $(p)$ or lattice $(l)$, which are found following the procedure presented in Ref. 22 . We arrive at the noninteracting (atomic) model by taking the limit where all Coulomb elements and population scattering rates are set to zero. The results presented here use a dot density of $N_{\mathrm{dot}}=5$ $\times 10^{14} \mathrm{~m}^{-2}$, a discretization of the WL into $100 k$ points, and a fixed lattice temperature of $200 \mathrm{~K}$, for which the literature ${ }^{1,23}$ gives scattering rates around $\gamma_{d}=1.5 \times 10^{12} \mathrm{~s}^{-1}$, $\gamma_{c-c}=2.0 \times 10^{12} \mathrm{~s}^{-1}, \quad \gamma_{c-p}=2.0 \times 10^{11} \mathrm{~s}^{-1}, \quad$ and $\quad \gamma_{\mathrm{nr}}=1.0$ $\times 10^{9} \mathrm{~s}^{-1}$.

\section{RESULTS}

The optical response for the three different schemes using a coupling field with an intensity of $26 \mathrm{MW} / \mathrm{cm}^{2}$ is shown in Fig. 2 illustrating both the interacting and the noninteracting cases. An immediate difference between the atomic and many-body approaches is the change in probe field energy toward negative detuning. This is due to the excitonic shift of the various probe transitions. An apparent feature of the ladder scheme [Fig. 2(a)] is that the peaks of the imaginary part of the susceptibility are highest for the many-body model. While the distance between the peaks remains the same, one can readily see that a larger area is covered by the manybody spectrum. This is evidence that oscillator strength has been shifted into the $|e 0\rangle-|h 0\rangle$ transition; in other words, it has been Coulomb enhanced. Considering the real part of the susceptibility, the enhancement of the probe transition results in more pronounced features, notably a larger slope at zero detuning. Both curves are generally shifted upward from the zero point; this behavior is due to transitions in the vicinity of the probe which are contributing to the background index of the area in question.

The optical responses for $V$ and $\Lambda$ schemes are shown in Figs. 2(b) and 2(c), respectively. For the interacting case the coupling field has been tuned to the zero density exciton resonance of the probe transition. The asymmetry in the peak heights of the imaginary part of the susceptibility has different origins for the interacting and noninteracting cases. The skewness in the atomic model is due to the fact that we are not dealing with a closed three-level system. The control field pumping the $|e 0\rangle-|h 0\rangle$ is also connecting the dipole allowed $|e 5\rangle-|h 5\rangle$ transition, however severely negatively detuned. Effectively we are dealing with two EIT schemes, the original $V(\Lambda)$ and a detuned $\Lambda(V)$ scheme. In general a negatively detuned $\Lambda$ or $V$ EIT scheme has a prominent shift in peak height toward positive detunings (resembling an optical Stark shift). What is seen in the two figures is an admixture of the symmetric peaks owing to the resonant $V(\Lambda)$ scheme and a Stark shifted transition. Further evidence of this effect has been obtained by altogether disallowing the "conflicting" transition in which case one recovers the symmetric result. In the models including interactions the same asymmetry should be expected but it is countered by a negative shift in resonance energy induced by the Coulomb interaction with the excited carriers. This means that the coupling is detuned positively with respect to the resonance, and hence the asymmetry tends toward negative probe energy. The probe transitions in these cases are not enhanced but rather they are suppressed by the inclusion of many-body effects; this can be seen by the fact that the features are generally smaller in magnitude than in the atomic model. The splitting of the peaks is larger although it shows that the effective Rabi frequency is higher, owing to the Coulomb enhancement of the $|e 0\rangle-|h 0\rangle$ coupling transition.

As a basis for comparing the slow light capabilities of the different schemes, we examine the maximum obtainable slowdown factor $S$, which is equal to the group index and is a measure of the group-velocity reduction. The slowdown factor $S$ is a figure of merit relevant for optical storage, and is defined via

$$
S=\frac{c_{0}}{v_{g}}=n+\omega \frac{\partial n}{\partial \omega},
$$

where $c_{0}$ is the speed of light in vacuum, and $n=\operatorname{Re}\left\{\left[n_{b}^{2}\right.\right.$ $\left.+\chi(\omega)]^{(1 / 2)}\right\}$ is the refractive index. The maximum slowdown is found at the frequency for which the slope of the refractive index is largest. Notice that the slowdown factor obtained away from resonance is given by the background refractive index. To make a just comparison, we detune the coupling field used in the many-body $V$ and $\Lambda$ models from the zero density exciton resonance so that the peaks of $\operatorname{Im}\{\chi(\omega)\}$ become symmetric. However, the amount of detuning for this to be realized depends on the intensity of the coupling field. 

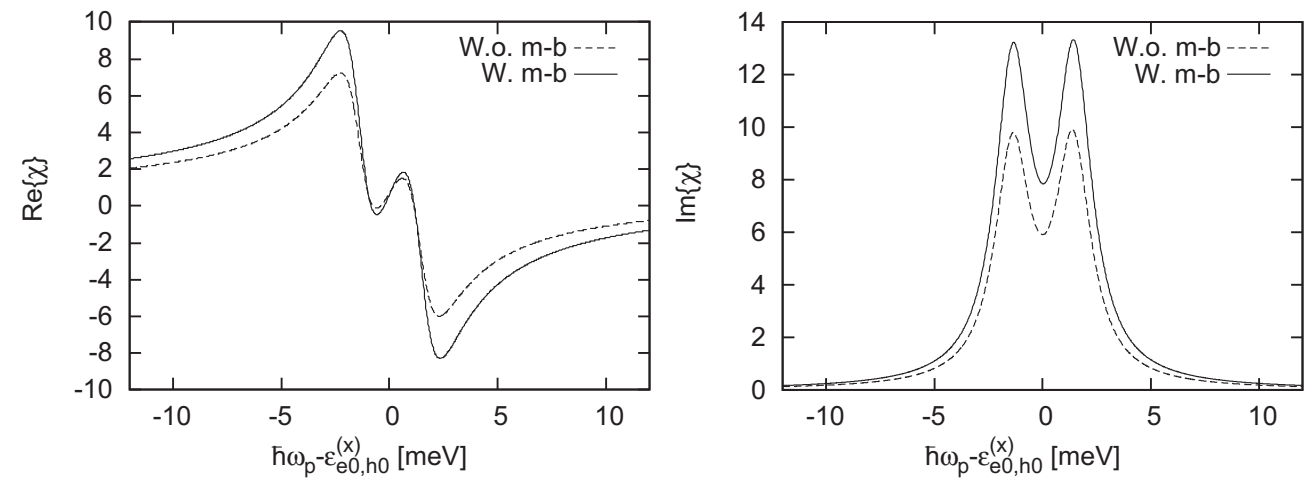

(a) Ladder scheme
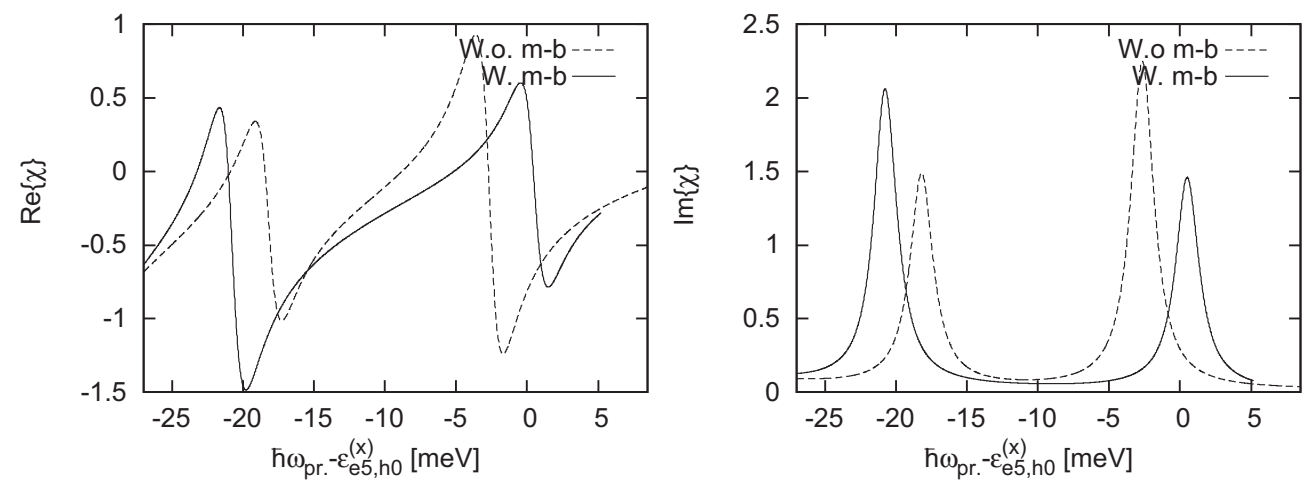

(b) $V$ scheme
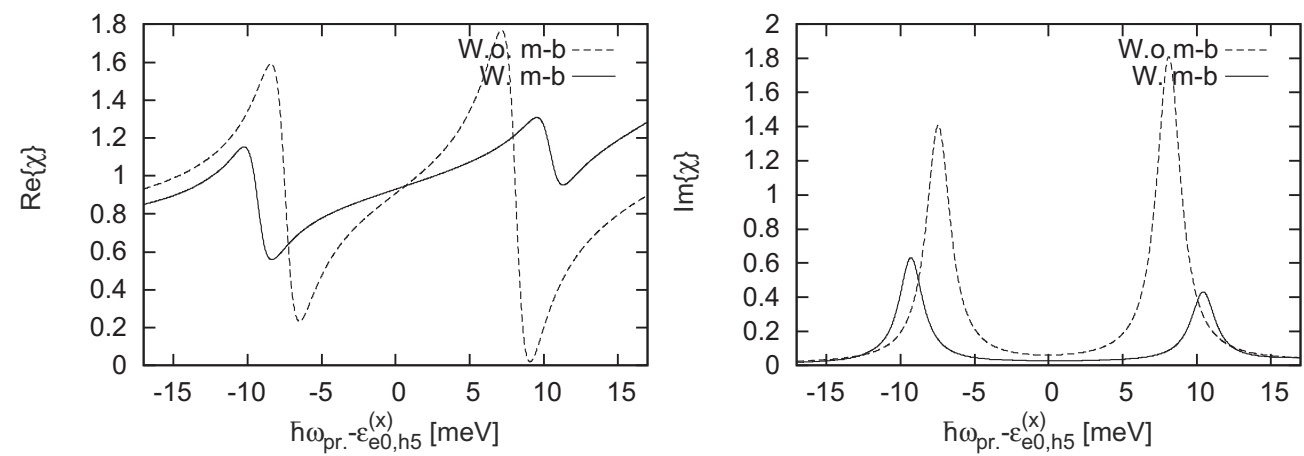

(c) $\Lambda$ scheme

FIG. 2. Ladder, $V$, and $\Lambda$ schemes using a coupling field intensity of $26 \mathrm{MW} / \mathrm{cm}^{2}$ : complex susceptibility vs probe energy. The dashed line corresponds to the atomic model, i.e., without many-body interactions, while the solid line is evaluated with many-body interactions. The energy is measured relative to the zero density excitonic resonances $\epsilon_{e 0, h 0}^{(x)}, \epsilon_{e 5, h 0}^{(x)}$, and $\epsilon_{e 0, h 5}^{(x)}$, respectively. To facilitate a comparison between the two cases, the noninteracting spectra have been shifted accordingly.

Changing the intensity in turn changes the amount of carriers being excited and thus the excitonic shift, which results in different detunings for different intensities.

Figure 3 depicts the maximum achievable slowdown factor and the corresponding absorption coefficient $\alpha(\omega)$ $=2 \frac{\omega}{c_{0}} \operatorname{Im}\{n(\omega)\}$ as a function of coupling power for all three schemes. A striking feature of Fig. 3 is that the inclusion of many-body effects leads to different results depending on the choice of EIT scheme. The results of the carrier-exciting $V$ and $\Lambda$ setups are seen to differ fundamentally from the ladder scheme. Inspecting the absorption coefficient plot we see two plateaus, corresponding to the maximum (minimum) absorption in the absence (presence) of EIT. The transition from the upper to the lower plateau happens across fewer orders of magnitude in the coupling power for the ladder scheme than the other two schemes. Here the absorption co- 

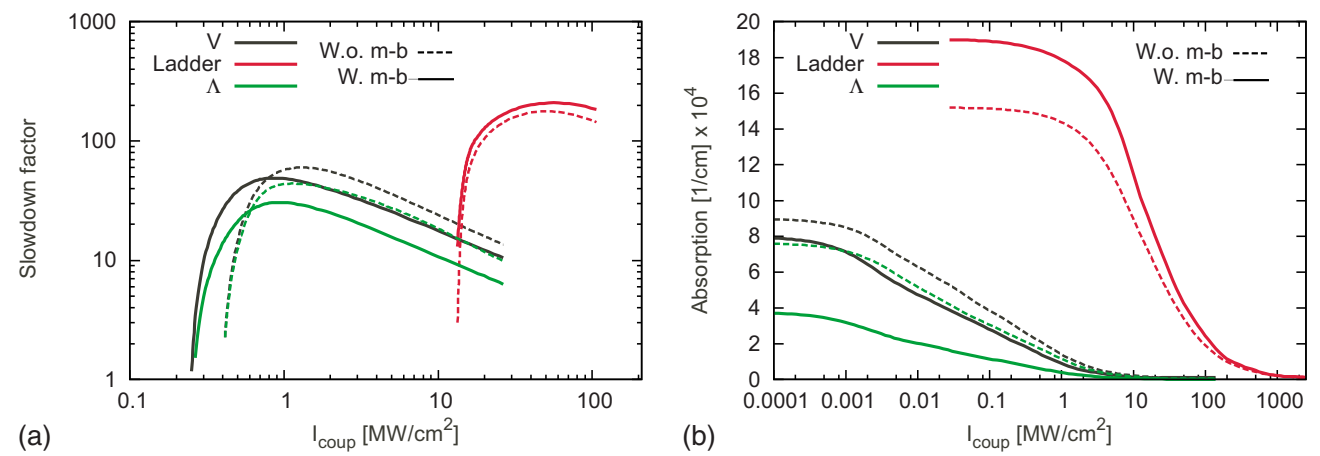

FIG. 3. (Color online) Maximum slowdown factor and corresponding absorption coefficient vs coupling intensity for various EIT schemes. The dashed curves are for the independent-particle (atomic) model while the solid curves include many-body interactions.

efficient drop is solely due to the quantum coherence effect setting in, whereas the transition for $\Lambda$ and $V$ happens across a significantly larger relative range. At low coupling power the absorption drop is driven by the excitation of carriers occupying the probe transition. As evidenced by the slowdown plot, the EIT effect sets in at larger coupling powers, only when we are near the lower plateau. The largest slowdown values are achieved using the ladder scheme, for which the slowdown factor is increased significantly when interactions are included. This is due to Coulomb enhancement of the $|e 0\rangle-|h 0\rangle$ resonance probed in this scheme. The slowdown effect is seen to disappear at the same value of coupling power for both cases, which indicates that the coupling transition $(|e 0\rangle-|e 5\rangle)$ utilized in the ladder configuration is unchanged by the inclusion of many-body effects. On the contrary, for the carrier-exciting schemes $V$ and $\Lambda$, manybody effects have a significant impact on the coupling threshold.

Both $V$ and $\Lambda$ schemes show largest slowdown values for the noninteracting model. On the other hand, the noninteracting model overestimates the minimum required coupling power for observing slow light by roughly a factor of two compared to the more realistic case of interacting particles. As both schemes utilize the same coupling transition, they experience the same coupling power threshold, in both the interacting as well as the noninteracting cases. The shift in required coupling power can be attributed solely to the Rabi energy enhancement of the coupling transition. This conclusion is reached by inspecting the absorption spectrum in the absence of a coupling field. By comparing the height of the $|e 0\rangle-|h 0\rangle$ resonance (coupling transition used in both schemes) with and without interactions, we find that the dipole moment of the transition is enlarged by roughly a factor of 1.2. If we, in the atomic model, enlarge the coupling dipole moment by the same amount, we end up with a result having the same minimal requirement on coupling power as the interacting case. This result stands in contrast to the findings in Ref. 10, where a shift in required coupling power, due to Coulomb enhancement, of two orders of magnitude was reported. However, this work was performed in a transient regime and a direct comparison is therefore not applicable here.

The $V$ scheme is preferable to the $\Lambda$ scheme due to its higher slowdown values. The reason is twofold. First, based on observations from the absorption spectrum without cou- pling, we find that the two probe transitions are both Coulomb suppressed; however, the $\Lambda$ scheme to a higher degree than $V$. Second the fact that the $V$ probe connects a hole ground state to an electron excited-state results in a larger Pauli blocking factor [the third term in Eq. (2)] as compared to the $\Lambda$ scheme. Figure 4 demonstrates this for the interacting case utilizing a coupling intensity of $2.5 \mathrm{MW} / \mathrm{cm}^{2}$. For the interacting model the redistribution of carriers plays a crucial role: the smaller effective electron mass leads to a larger energy spacing of the electronic levels, which means that the electron excited states become less populated than their hole counterparts. In the case presented here the hole ground state is seen to be depleted and carriers are redistributed into the higher lying energy states. For electrons the redistribution is less prominent, and as the coupling field excites more and more carriers, electrons accumulate in the ground state. Thus the Pauli blocking factor seen by the probe in the $V$ configuration is always the larger, which ultimately translates into an increased slowdown factor. This result is quite general and could act as a pointer for experimental realization of EIT mediated slow light.

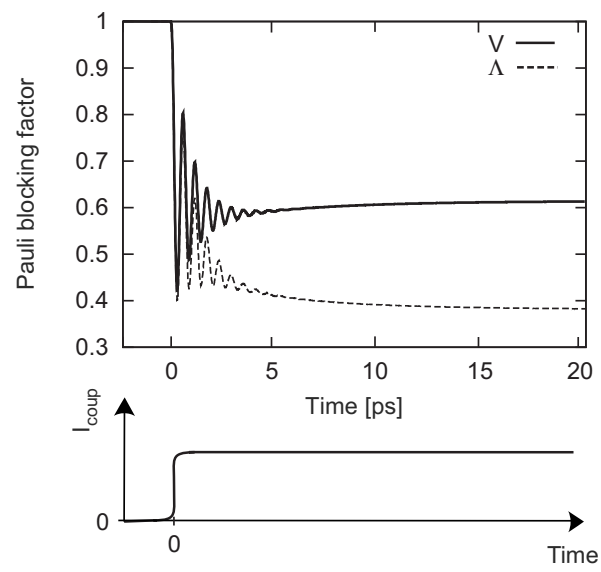

FIG. 4. Temporal development of the Pauli probe blocking factors for $\Lambda$ and $V$ schemes at coupling intensity of $2.5 \mathrm{MW} / \mathrm{cm}^{2}$. Shown in the figure are the Rabi oscillations of the populations at the onset of the coupling field and the relaxation toward their stationary value. Also shown is an illustration of the "turn on" of the coupling laser. 


\section{CONCLUSION}

In conclusion, we have investigated the slow light properties of InAs QDs using a model including many-body effects for three different EIT schemes and found fundamental differences. The ladder scheme that utilizes a transparent coupling transition has its slowdown factor increased due to Coulomb enhancement. However, there is observed no change in the necessary coupling power required to reach EIT. Conversely in the $V$ and $\Lambda$ schemes, many-body effects enhance the coupling transition resulting in a lowering of the necessary coupling power. The $V$ type configuration is found to be preferable due to a favorable redistribution of carriers.

\section{ACKNOWLEDGMENTS}

This work has been supported by the Danish Research Council for Technology and Innovation through the project QUEST and the European Commission through the IST project "QPhoton" (Contract No. IST-29283). A.P.J. is grateful to the FiDiPro program of the Finnish Academy during the final stages of this work. *jakob.houmark@nanotech.dtu.dk

${ }^{1}$ P. Borri, W. Langbein, S. Schneider, U. Woggon, R. L. Sellin, D. Ouyang, and D. Bimberg, Phys. Rev. Lett. 87, 157401 (2001).

${ }^{2}$ J. Mørk, F. Öhman, M. van der Poel, Y. Chen, P. Lunnemann, and K. Yvind, Laser Photonics Rev. 3, 30 (2009).

${ }^{3}$ C. J. Chang-Hasnain, P. C. Ku, J. Kim, and S. L. Chuang, Proc. IEEE 91, 1884 (2003)

${ }^{4}$ S. E. Harris, J. E. Field, and A. Imamoglu, Phys. Rev. Lett. 64, 1107 (1990).

${ }^{5}$ S. E. Harris, Phys. Today 50, 36 (1997).

${ }^{6}$ S. Marcinkevičius, A. Gushterov, and J. P. Reithmaier, Appl. Phys. Lett. 92, 041113 (2008).

${ }^{7}$ J. Kim, S. L. Chuang, P. C. Ku, and C. J. Chang-Hasnain, J. Phys.: Condens. Matter 16, S3727 (2004).

${ }^{8}$ P. Janes, J. Tidstrom, and L. Thylen, J. Lightwave Technol. 23, 3893 (2005).

${ }^{9}$ P. K. Nielsen, H. Thyrrestrup, J. Mørk, and B. Tromborg, Opt. Express 15, 6396 (2007).

${ }^{10}$ S. Michael, W. W. Chow, and H. C. Schneider, Appl. Phys. Lett. 89, 181114 (2006)

${ }^{11}$ W. W. Chow, S. Michael, and H. C. Schneider, J. Mod. Opt. 54, 2413 (2007)

${ }^{12}$ M. S. Bigelow, N. N. Lepeshkin, and R. W. Boyd, Science 301,
$200(2003)$

${ }^{13}$ S. W. Chang and S. L. Chuang, Phys. Rev. B 72, 235330 (2005).

${ }^{14}$ J. Houmark, T. R. Nielsen, J. Mørk, and A. P. Jauho, J. Phys.: Conf. Ser. 107, 012005 (2008).

${ }^{15}$ R. V. N. Melnik and M. Willatzen, Nanotechnology 15, 1 (2004).

${ }^{16}$ J. Houmark, D. Barettin, B. Lassen, and M. Willatzen (unpublished).

${ }^{17} \mathrm{H}$. Haug and S. W. Koch, Quantum Theory of the Optical and Electronic Properties of Semiconductors, 3rd ed. (World Scientific, Singapore, 1990).

${ }^{18}$ T. R. Nielsen, Ph.D. thesis, Institute for Theoretical Physics, University of Bremen, Germany, 2005; URL: http:// elib.suub.unibremen.de/diss/docs/00010001.pdf.

${ }^{19}$ T. R. Nielsen, P. Gartner, and F. Jahnke, Phys. Rev. B 69 235314 (2004).

${ }^{20}$ T. R. Nielsen, P. Gartner, M. Lorke, J. Seebeck, and F. Jahnke, Phys. Rev. B 72, 235311 (2005).

${ }^{21}$ J. Mørk and A. Mecozzi, J. Opt. Soc. Am. B 13, 1803 (1996).

${ }^{22}$ W. W. Chow, H. C. Schneider, and M. C. Phillips, Phys. Rev. A 68, 053802 (2003).

${ }^{23}$ P. Borri, W. Langbein, J. Mork, J. M. Hvam, F. Heinrichsdorff, M. H. Mao, and D. Bimberg, Phys. Rev. B 60, 7784 (1999). 